ANNALES

POLONICI MATHEMATICI

$97.1(2010)$

\title{
Invariance of regularity conditions under definable, locally Lipschitz, weakly bi-Lipschitz mappings
}

\author{
by Małgorzata Czapla (Kraków)
}

\begin{abstract}
We describe the notion of a weakly Lipschitz mapping on a $C^{q}$ stratification. We also distinguish a class of regularity conditions that are in some sense invariant under definable, locally Lipschitz and weakly bi-Lipschitz homeomorphisms. This class includes the Whitney (B) condition and the Verdier condition.
\end{abstract}

Introduction. The first goal of this paper is to study the notion of a weakly Lipschitz mapping on a fixed $C^{q}$ stratification, which generalizes the notion of a Lipschitz function. Section 1 consists of the basic definitions and notation, while in Section 2 we introduce the main idea together with its geometrical interpretation and discuss its fundamental properties. Weakly Lipschitz mappings were used earlier under the name fonctions rugueuses by J.-L. Verdier [Ver, who considered them from a different point of view.

The second goal of this paper is to distinguish a special class of regularity conditions (Section 3); namely, the conditions which are definable, generic, $C^{q}$ invariant and have the lifting property with respect to locally Lipschitz mappings and the projection property with respect to weakly Lipschitz mappings. In the definable case these properties make the regularity conditions in some sense invariant with respect to definable, locally Lipschitz, weakly bi-Lipschitz homeomorphisms (Theorem 3.15).

In Sections 4 and 5 we prove that the Whitney (B) condition and the Verdier condition belong to the above class.

1. Preliminaries. We denote by $|\cdot|$ the euclidean norm of $\mathbb{R}^{n}$, and $S^{n-1}=\left\{x \in \mathbb{R}^{n}:|x|=1\right\}$. In the whole paper $q$ denotes the class of smoothness of a mapping, so $q \in \mathbb{N}$ or $q \in\{\infty, \omega\}$, unless otherwise indicated.

2010 Mathematics Subject Classification: Primary 14P10, 32B20; Secondary 32S60, 51N20, $51 \mathrm{~F} 99$.

Key words and phrases: o-minimal structure, weakly Lipschitz mapping, Verdier condition, Whitney conditions. 
Also we denote by $\mathbb{G}_{k, n}$ the Grassmann manifold of $k$-dimensional vector subspaces of $\mathbb{R}^{n}$. Then $\mathbb{P}_{n-1}=\mathbb{G}_{1, n}$ is the real projective space of dimension $n-1$.

Definition 1.1. Let $v \in S^{n-1}$ and $W$ be a nonzero linear subspace of $\mathbb{R}^{n}$. We put

$$
d(v, W)=\inf \left\{\sin (v, w): w \in W \cap S^{n-1}\right\},
$$

where $\sin (v, w)$ denotes the sine of the angle between the vectors $v$ and $w$. We also put $d(u, W)=1$ if $W=\{0\}$.

Definition 1.2. For any $P \in \mathbb{G}_{k, n}$ and $Q \in \mathbb{G}_{l, n}$, we put

$$
d(P, Q)=\sup \left\{d(\lambda, Q): \lambda \in P \cap S^{n-1}\right\}
$$

when $k>0$, and $d(P, Q)=0$ when $k=0$.

Now we list some elementary properties of the function $d$, leaving the proof to the reader.

Proposition 1.3.

(a) $0 \leq d(P, Q) \leq 1$.

(b) $d(P, Q)=0 \Leftrightarrow P \subset Q$.

(c) $d(P, Q)=1 \Leftrightarrow P \cap Q^{\perp} \neq\{0\}$ (or $\left.d(P, Q)<1 \Leftrightarrow P \cap Q^{\perp}=\{0\}\right)$.

(d) $d(P, R) \leq d(P, Q)+d(Q, R)$.

(e) $d(P, Q)=d(Q, P)$ if $\operatorname{dim} P=\operatorname{dim} Q$.

(f) $d$ is a metric on every $\mathbb{G}_{k, n}$.

(g) We have

$$
\begin{aligned}
d(\mathbb{R} v, Q) & =\left|v-\pi_{Q}(v)\right|=\operatorname{dist}(v, Q)=\left|\pi_{Q^{\perp}}(v)\right| \\
& =\sin \left(v, \frac{\pi_{Q}(v)}{\left|\pi_{Q}(v)\right|}\right)=d\left(\mathbb{R} v, \mathbb{R} \frac{\pi_{Q}(v)}{\left|\pi_{Q}(v)\right|}\right),
\end{aligned}
$$

where $\pi_{Q}$ denotes the orthogonal projection onto $Q, v$ is a unit vector not orthogonal to $Q$, and $\operatorname{dist}(v, Q)=\inf \{|v-w|: w \in Q\}$.

(h) Consider the following metric on $\mathbb{P}_{n-1}$ :

$$
\widetilde{d}(\mathbb{R} v, \mathbb{R} w)=\min \{|u-w|,|u+w|\} \quad \text { for } u, w \in S^{n-1} .
$$

Then

$$
\frac{1}{\sqrt{2}} \widetilde{d}(\mathbb{R} v, \mathbb{R} w) \leq d(\mathbb{R} v, \mathbb{R} w) \leq \widetilde{d}(\mathbb{R} v, \mathbb{R} w) .
$$

(i) If $P \subset P^{\prime}$, then $d(P, Q) \leq d\left(P^{\prime}, Q\right)$.

(j) If $Q^{\prime} \subset Q$, then $d(P, Q) \leq d\left(P, Q^{\prime}\right)$.

To transform $d$ into a metric $D$ in $\mathbb{G}_{n}=\bigcup_{k=1}^{n-1} \mathbb{G}_{k, n}$ (disjoint union), we put

$$
D(P, Q)=\max \{d(P, Q), d(Q, P)\} .
$$


Then $\mathbb{G}_{k, n}$ are open-closed components in $\mathbb{G}_{n}$ and $D\left(\mathbb{G}_{k, n}, \mathbb{G}_{l, n}\right)=1$ for $k \neq l$. It is easy to check that

$$
\left|d\left(P_{1}, Q_{1}\right)-d\left(P_{2}, Q_{2}\right)\right| \leq D\left(P_{1}, P_{2}\right)+D\left(Q_{1}, Q_{2}\right),
$$

hence the function $d$ is continuous.

We will need another function which characterizes the mutual position of two linear subspaces $V$ and $W$ of $\mathbb{R}^{n}$.

Definition 1.4. Set

$$
\delta(V, W)= \begin{cases}\inf \left\{d(v, W): v \in V \cap S^{n-1}\right\} & \text { if } V \neq\{0\} \\ 1 & \text { if } V=\{0\} .\end{cases}
$$

The reader will easily check the following properties:

Proposition 1.5.

(i) $\delta(V, W)=0 \Leftrightarrow V \cap W \neq\{0\}$.

(ii) $\delta(V, W)>0 \Leftrightarrow V \cap W=\{0\}$.

(iii) $\delta(V, W)=1 \Leftrightarrow V \perp W$.

(iv) $\delta(V, W) \leq d(V, W) \leq D(V, W)$ if $V \neq\{0\} \neq W$.

(v) $\delta$ is continuous.

Proposition 1.6. Let $\Lambda \subset \mathbb{R}^{n}$ be a $C^{q}$ submanifold and $f: \Lambda \rightarrow \mathbb{R}^{m}$ be a $C^{q}$ mapping. Assume that for each $\left.x \in \operatorname{graph} f\right|_{\Lambda}$,

$$
\delta\left(T_{x} \text { graph }\left.f\right|_{\Lambda},\{0\} \times \mathbb{R}^{m}\right) \geq \alpha>0,
$$

where $\alpha$ is a positive constant. Then

(i) For any $x_{0} \in \overline{\left.\operatorname{graph} f\right|_{\Lambda}}$ and any sequence $\left\{x_{\nu}\right\}_{\nu \in \mathbb{N}} \subset$ graph $\left.f\right|_{\Lambda}$ converging to $x_{0}$ such that the sequence $\left\{T_{x_{\nu}} \text { graph }\left.f\right|_{\Lambda}\right\}_{\nu \in \mathbb{N}}$ is convergent,

$$
\delta\left(\left.\lim _{\nu \rightarrow \infty} T_{x_{\nu}} \operatorname{graph} f\right|_{\Lambda},\{0\} \times \mathbb{R}^{m}\right) \geq \alpha>0 .
$$

(ii) For any $C^{q}$ submanifold $M \subset \Lambda, M \times \mathbb{R}^{m}$ is transversal to graph $\left.f\right|_{\Lambda}$ in $\Lambda \times \mathbb{R}^{n}$.

Proof. Observe that (i) follows from the continuity of $\delta$.

(ii) By Proposition 1.5(ii),

$$
\left.T_{x} \operatorname{graph} f\right|_{\Lambda} \cap\left(\{0\} \times \mathbb{R}^{m}\right)=\{0\},
$$

hence it is enough to observe that $\left.\operatorname{dim} T_{x} \operatorname{graph} f\right|_{\Lambda}=\operatorname{dim} \Lambda$.

Proposition 1.7. Let $\Lambda \subset \mathbb{R}^{n}$ be a $C^{q}$ submanifold and let $f: \Lambda \rightarrow \mathbb{R}^{m}$ be a Lipschitz $C^{q}$ mapping. Then there exists a positive constant $\alpha$ such that

$$
\delta\left(T_{x} \operatorname{graph} f,\{0\} \times \mathbb{R}^{m}\right) \geq \alpha>0
$$

for each $x \in \operatorname{graph} f$. 
Proof. There exists $L>0$ such that $\left\|d_{y} f\right\| \leq L$ for each $y \in \Lambda$. Now let $v \in T_{x}$ graph $f$ with $|v|=1$ for some $x=(y, f(y)), y \in \Lambda$. Then there exists a vector $\widetilde{v} \in T_{y} \Lambda$ such that

$$
v=\left(\widetilde{v}, d_{y} f(\widetilde{v})\right) .
$$

Hence

$$
1=|v|=\sqrt{|\widetilde{v}|^{2}+\left|d_{y} f(\widetilde{v})\right|^{2}} \leq \sqrt{1+L^{2}}|\widetilde{v}| .
$$

Therefore

$$
|\widetilde{v}| \geq \frac{1}{\sqrt{1+L^{2}}}>0 .
$$

On the other hand, $d\left(v,\{0\} \times \mathbb{R}^{n}\right)=|\widetilde{v}|$.

Now we briefly recall the notion of a $C^{q}$ stratification.

Definition 1.8. Let $A$ be a subset of $\mathbb{R}^{n}$. A $C^{q}$ stratification of $A$ is a (locally) finite family $\mathfrak{X}_{A}$ of connected $C^{q}$ submanifolds of $\mathbb{R}^{n}$ (called strata) such that

- $A=\bigcup \mathfrak{X}_{A}$;

- if $\Gamma_{1}, \Gamma_{2} \in \mathfrak{X}_{A}, \Gamma_{1} \neq \Gamma_{2}$ then $\Gamma_{1} \cap \Gamma_{2}=\emptyset$;

- for each $\Gamma \in \mathfrak{X}_{A}$ the set $(\bar{\Gamma} \backslash \Gamma) \cap A$ is a union of some strata from $\mathfrak{X}_{A}$ of dimension $<\operatorname{dim} \Gamma$.

We say that the stratification $\mathfrak{X}_{A}$ is compatible with a family of sets $B_{i} \subset A$, $i \in I$, if every set $B_{i}$ is a union of some strata of $\mathfrak{X}_{A}$.

Actually, we will only be interested in finite stratifications.

Definition 1.9. Let $A \subset \mathbb{R}^{n}$, let $f: A \rightarrow \mathbb{R}^{m}$ be a continuous mapping, and let $\mathfrak{X}_{A}$ be a $C^{q}$ stratification of $A$ such that $\left.f\right|_{\Gamma}$ is of class $C^{q}$ for all $\Gamma \in \mathfrak{X}_{A}$. Then the induced $C^{q}$ stratification of graph $f$ is

$$
\mathfrak{X}_{\text {graph } f}\left(\mathfrak{X}_{A}\right)=\left\{\text { graph }\left.f\right|_{\Gamma}: \Gamma \in \mathfrak{X}_{A}\right\} \text {. }
$$

A natural setting for our results is the theory of o-minimal structures (or more generally geometric categories), as presented in [D] (or [DM]). In the whole paper the adjective definable (e.g. definable subset, definable mapping) will refer to any fixed o-minimal structure on the ordered field $\mathbb{R}$ of real numbers.

2. Weakly Lipschitz mappings. In this section we define weakly Lipschitz mappings and list their important properties.

Definition 2.1. Let $A$ be a subset of $\mathbb{R}^{n}$ and let $\mathfrak{X}_{A}$ be a finite $C^{q}$ stratification of $A$.

We say that a mapping $f: A \rightarrow \mathbb{R}^{m}$ is weakly Lipschitz of class $C^{q}$ on the stratification $\mathfrak{X}_{A}$ if for each stratum $\Gamma \in \mathfrak{X}_{A}$ the restriction $\left.f\right|_{\Gamma}$ is of class $C^{q}$ and the pair $\left(f, \mathfrak{X}_{A}\right)$ satisfies one of the following equivalent conditions: 
(a) Whenever $\Lambda, \Gamma \in \mathfrak{X}_{A}, \Gamma \subset \bar{\Lambda} \backslash \Lambda, a \in \Gamma$ and $\left\{a_{\nu}\right\}_{\nu \in \mathbb{N}},\left\{b_{\nu}\right\}_{\nu \in \mathbb{N}}$ are sequences such that $a_{\nu} \in \Gamma$ and $b_{\nu} \in \Lambda$ for $\nu \in \mathbb{N}$, then

$$
a_{\nu}, b_{\nu} \rightarrow a(\nu \rightarrow \infty) \Rightarrow \limsup _{\nu \rightarrow \infty} \frac{\left|f\left(a_{\nu}\right)-f\left(b_{\nu}\right)\right|}{\left|a_{\nu}-b_{\nu}\right|}<\infty .
$$

(b) For any stratum $\Gamma \in \mathfrak{X}_{A}$ and any $a \in \Gamma$ there exists a neighbourhood $U_{a}$ of $a$ such that the mapping

$$
\psi:\left(\Gamma \cap U_{a}\right) \times\left((A \backslash \Gamma) \cap U_{a}\right) \ni(x, y) \mapsto \frac{|f(x)-f(y)|}{|x-y|} \in \mathbb{R}
$$

is bounded.

(c) For any strata $\Lambda, \Gamma \in \mathfrak{X}_{A}$ with $\Gamma \subset \bar{\Lambda} \backslash \Lambda$ and for any $a \in \Gamma$ there exists a neighbourhood $U_{a}$ of $a$ such that the mapping

$$
\psi:\left(\Gamma \cap U_{a}\right) \times\left(\Lambda \cap U_{a}\right) \ni(x, y) \mapsto \frac{|f(x)-f(y)|}{|x-y|} \in \mathbb{R}
$$

is bounded.

(d) Whenever $\Lambda, \Gamma \in \mathfrak{X}_{\text {graph } f}\left(\mathfrak{X}_{A}\right), \Gamma \subset \bar{\Lambda} \backslash \Lambda, x \in \Gamma$ and $\left\{x_{\nu}\right\}_{\nu \in \mathbb{N}} \subset \Gamma$, $\left\{y_{\nu}\right\}_{\nu \in \mathbb{N}} \subset \Lambda$ are sequences convergent to $x$, then

$$
d\left(\lim _{\nu \rightarrow \infty} \mathbb{R}\left(x_{\nu}-y_{\nu}\right),\{0\} \times \mathbb{R}^{m}\right)>0 .
$$

(e) Whenever $\Lambda, \Gamma \in \mathfrak{X}_{\operatorname{graph} f}\left(\mathfrak{X}_{A}\right), \Gamma \subset \bar{\Lambda} \backslash \Lambda, x \in \Gamma$, and $\left\{x_{\nu}\right\}_{\nu \in \mathbb{N}} \subset \Gamma$, $\left\{y_{\nu}\right\}_{\nu \in \mathbb{N}} \subset \Lambda$ are sequences convergent to $x$ such that the limit

$$
L=\lim _{\nu \rightarrow \infty} \mathbb{R}\left(x_{\nu}-y_{\nu}\right)
$$

exists, then $L \cap\left(\{0\} \times \mathbb{R}^{m}\right)=\{0\}$.

Proposition 2.2. The conditions (a)-(e) from Definition 2.1 are equivalent.

Proof. (a) $\Rightarrow$ (c). Suppose that (c) is not satisfied. Then we find some strata $\Gamma, \Lambda \in \mathfrak{X}_{A}$ with $\Gamma \subset \bar{\Lambda} \backslash \Lambda$ and a point $a \in \Gamma$ for which we can find a basis of neighbourhoods $\left\{U_{n}\right\}_{n \in \mathbb{N}}$ and two sequences $\left\{a_{n}\right\}_{n \in \mathbb{N}},\left\{b_{n}\right\}_{n \in \mathbb{N}}$ such that $a_{n} \in \Gamma \cap U_{n}, b_{n} \in \Lambda \cap U_{n}$ and

$$
\frac{\left|f\left(a_{n}\right)-f\left(b_{n}\right)\right|}{\left|a_{n}-b_{n}\right|}>n,
$$

a contradiction with the assumption.

$(\mathrm{c}) \Rightarrow(\mathrm{a})$ and $(\mathrm{b}) \Leftrightarrow(\mathrm{c})$ are trivial.

(a) $\Leftrightarrow(\mathrm{d}) \Leftrightarrow(\mathrm{e})$. Since $\Gamma=\left.\operatorname{graph} f\right|_{\Gamma^{\prime}}, \Lambda=\left.\operatorname{graph} f\right|_{\Lambda^{\prime}}, x=(a, f(a))$, $x_{\nu}=\left(a_{\nu}, f\left(a_{\nu}\right)\right)$ and $y_{\nu}=\left(b_{\nu}, f\left(b_{\nu}\right)\right)$, when $\Gamma^{\prime}, \Lambda^{\prime} \in \mathfrak{X}_{A}, a, a_{\nu} \in \Gamma^{\prime}, b_{\nu} \in \Lambda^{\prime}$, 
$a_{\nu} \rightarrow a$ and $b_{\nu} \rightarrow a(\nu \rightarrow \infty)$, it is enough to observe that

$$
\begin{aligned}
d\left(\mathbb{R}\left(x_{\nu}-y_{\nu}\right),\{0\} \times \mathbb{R}^{m}\right) & =\frac{\left|a_{\nu}-b_{\nu}\right|}{\left|\left(a_{\nu}, f\left(a_{\nu}\right)\right)-\left(b_{\nu}, f\left(b_{\nu}\right)\right)\right|} \\
& =\frac{1}{\sqrt{1+\left(\frac{\left|f\left(a_{\nu}\right)-f\left(b_{\nu}\right)\right|}{\left|a_{\nu}-b_{\nu}\right|}\right)^{2}}} .
\end{aligned}
$$

REMARK 2.3. If $f: A \rightarrow \mathbb{R}^{m}$ is weakly Lipschitz on a stratification $\mathfrak{X}_{A}$ of $A$, then $f$ is continuous on $A$.

Of course, the weak lipschitzianity is a generalization of the Lipschitz condition. Obviously, we have the following

Proposition 2.4. Let $A \subset \mathbb{R}^{n}$ and let $f: A \rightarrow \mathbb{R}^{m}$ be a locally Lipschitz mapping. Assume that $A$ admits a $C^{q}$ stratification $\mathfrak{X}_{A}$ such that for all strata $\Gamma \in \mathfrak{X}_{A}$ the map $\left.f\right|_{\Gamma}$ is of class $C^{q}$. Then $f$ is weakly Lipschitz of class $C^{q}$ on $\mathfrak{X}_{A}$.

By the $C^{q}$ Cell Decomposition Theorem (see [DM]), we have the following

Corollary 2.5. Let $A \subset \mathbb{R}^{n}$ and let $f: A \rightarrow \mathbb{R}^{m}$ be a definable locally Lipschitz mapping. Then there exists a definable $C^{q}$ stratification $\mathfrak{X}_{A}$ of $A$ such that $f$ is weakly Lipschitz of class $C^{q}$ on $\mathfrak{X}_{A}$.

The weak lipschitzianity is a much weaker property than the local Lipschitz condition, as shown by the examples below.

Example 2.6. Let $A \subset \mathbb{R}^{2}, A=\Lambda \cup \Gamma_{1} \cup \Gamma_{2}$ and $\mathfrak{X}_{A}=\left\{\Lambda, \Gamma_{1}, \Gamma_{2}\right\}$, where

$$
\begin{aligned}
\Lambda & =\left\{(x, y) \in \mathbb{R}^{2}: x \in(0,1), \frac{1}{2} x^{2}<y<x^{2}\right\}, \\
\Gamma_{1} & =\left\{(x, y) \in \mathbb{R}^{2}: x \in(0,1), y=\frac{1}{2} x^{2}\right\}, \quad \Gamma_{2}=\{(0,0)\} .
\end{aligned}
$$

The mapping

$$
f: A \ni(x, y) \mapsto(x, \sqrt{y}) \in \mathbb{R}^{2}
$$

is not Lipschitz in any neighbourhood of $(0,0)$, because $\frac{\partial f}{\partial y}=\left(0, \frac{1}{2 \sqrt{y}}\right)$ is unbounded. However, $f$ is weakly Lipschitz, because it is locally Lipschitz on $A \backslash\{(0,0)\}$ and

$$
\frac{|f(x, y)|}{|(x, y)|}=\sqrt{\frac{x^{2}+y}{x^{2}+y^{2}}} \leq \sqrt{\frac{2 x^{2}}{x^{2}}} \leq \sqrt{2} .
$$

EXAmple 2.7. Let $\Lambda=\left\{(x, y) \in \mathbb{R}^{2}: y>0\right\}, \Gamma=\left\{(x, y) \in \mathbb{R}^{2}: y=0\right\}$, $A=\Lambda \cup \Gamma$. Define $f: A \rightarrow \mathbb{R}$ by

$$
f(x, y)= \begin{cases}\left(x^{2} / y^{7}-y^{2}\right)^{2}, & 0<x^{2}<y^{9} \\ 0, & x^{2} \geq y^{9} \geq 0\end{cases}
$$


Then $f$ is weakly Lipschitz of class $C^{1}$ on $\{\Lambda, \Gamma\}$. Indeed, $f$ is $C^{1}$ on $A \backslash\{(0,0)\}$, hence locally Lipschitz on $A \backslash\{(0,0)\}$. Moreover, if $(x, y) \in \Lambda$, $\left(x^{\prime}, 0\right) \in \Gamma$ and $f(x, y) \neq 0$, then

$$
\frac{\left|f(x, y)-f\left(x^{\prime}, 0\right)\right|}{\left|(x, y)-\left(x^{\prime}, 0\right)\right|}=\frac{\left(x^{2} / y^{7}-y^{2}\right)^{2}}{\sqrt{\left(x-x^{\prime}\right)^{2}+y^{2}}} \leq \frac{y^{4}}{y}=y^{3} \text {. }
$$

Nevertheless, $f$ is not a Lipschitz mapping in any neighbourhood of $(0,0)$, because

$$
\left|\frac{\partial f}{\partial x}\left(\frac{y^{9 / 2}}{\sqrt{3}}, y\right)\right|=\frac{8}{3 \sqrt{3}} \frac{1}{\sqrt{y}} \rightarrow \infty \quad \text { as } y \rightarrow 0 .
$$

The proofs of the following three propositions are straightforward.

Proposition 2.8. Let $A \subset \mathbb{R}^{n}$ and let $f: A \rightarrow \mathbb{R}^{n}$ be weakly Lipschitz of class $C^{q}(q \geq 1)$ on a $C^{q}$ stratification $\mathfrak{X}_{A}$. Let $B \subset A$. Then for any $C^{q}$ stratification $\mathfrak{X}_{B}$ of $B$, compatible with $\mathfrak{X}_{A}$, the mapping $f$ is weakly Lipschitz of class $C^{q}$ on $\mathfrak{X}_{B}$.

Proposition 2.9. Let $f: A \rightarrow \mathbb{R}^{p}$ be a weakly Lipschitz mapping of class $C^{q}(q \geq 1)$ on a $C^{q}$ stratification $\mathfrak{X}_{A}$ of $A \subset \mathbb{R}^{n}$, and let $g: B \rightarrow \mathbb{R}^{r}$ be a weakly Lipschitz mapping of class $C^{q}$ on a $C^{q}$ stratification $\mathfrak{X}_{B}$ of $B \subset \mathbb{R}^{p}$. Assume that the image under $f$ of each stratum from $\mathfrak{X}_{A}$ is contained in some stratum from $\mathfrak{X}_{B}$ (in particular, $f(A) \subset B$ ). Then $g \circ f: A \rightarrow \mathbb{R}^{r}$ is a weakly Lipschitz mapping of class $C^{q}$ on $\mathfrak{X}_{A}$.

REMARK 2.10. The last proposition allows one to define a category with stratified sets as objects and weakly Lipschitz mappings of class $C^{q}(q \geq 1)$ as morphisms.

Proposition 2.11. Let $f: A \rightarrow \mathbb{R}^{m}$ and $g: A \rightarrow \mathbb{R}^{p}$ be two weakly Lipschitz mappings of class $C^{q}$ on a $C^{q}$ stratification $\mathfrak{X}_{A}$ of $A \subset \mathbb{R}^{n}$. Then the mapping

$$
(f, g): A \ni x \mapsto(f(x), g(x)) \in \mathbb{R}^{m} \times \mathbb{R}^{p}
$$

is weakly Lipschitz of class $C^{q}$ on $\mathfrak{X}_{A}$ as well.

Definition 2.12. For a homeomorphic embedding $f: A \rightarrow \mathbb{R}^{m}$ and a $C^{q}$ stratification $\mathfrak{X}_{A}$ of $A$ such that for any $\Gamma \in \mathfrak{X}_{A}$ the map $\left.f\right|_{\Gamma}$ is a $C^{q}$ embedding, we have a natural $C^{q}$ stratification of the image $f(A)$,

$$
f \mathfrak{X}_{A}=\left\{f(\Gamma): \Gamma \in \mathfrak{X}_{A}\right\} .
$$

This leads to the following definition of a weakly bi-Lipschitz homeomorphism:

Definition 2.13. Let $A \subset \mathbb{R}^{n}$ and let $f: A \rightarrow \mathbb{R}^{m}$ be a homeomorphic embedding. Let $\mathfrak{X}_{A}$ be a $C^{q}$ stratification $(q \geq 1)$ of $A$ such that for all $\Gamma \in \mathfrak{X}_{A}$ the mapping $\left.f\right|_{\Gamma}$ is a $C^{q}$ embedding. 
We say that $f$ is weakly bi-Lipschitz of class $C^{q}$ on the stratification $\mathfrak{X}_{A}$ if $f$ is weakly Lipschitz of class $C^{q}$ on $\mathfrak{X}_{A}$ and the inverse $f^{-1}: f(A) \rightarrow A \subset \mathbb{R}^{n}$ is weakly Lipschitz of class $C^{q}$ on $f \mathfrak{X}_{A}$.

In order to check that the inverse mapping is weakly Lipschitz on $f \mathfrak{X}_{A}$, we can use the following obvious

Proposition 2.14. Let $A \subset \mathbb{R}^{n}$ and let $f: A \rightarrow \mathbb{R}^{m}$ be a homeomorphic embedding. Let $\mathfrak{X}_{A}$ be a $C^{q}$ stratification of $A$ and assume that for each stratum $\Gamma \in \mathfrak{X}_{A}$ the mapping $\left.f\right|_{\Gamma}$ is a $C^{q}$ embedding. Then the mapping $f^{-1}: f(A) \rightarrow A$ is weakly Lipschitz of class $C^{q}$ on the stratification $f \mathfrak{X}_{A}$ if and only if it satisfies the following condition:

( $\left.\mathrm{a}^{\prime}\right)$ for any strata $\Gamma, \Lambda \in \mathfrak{X}_{A}$ with $\Gamma \subset \bar{\Lambda} \backslash \Lambda$ and for any point $a \in \Gamma$, if $\left\{a_{\nu}\right\}_{\nu \in \mathbb{N}},\left\{b_{\nu}\right\}_{\nu \in \mathbb{N}}$ are sequences such that $a_{\nu} \in \Gamma$ and $b_{\nu} \in \Lambda$ for $\nu \in \mathbb{N}$, then

$$
a_{\nu}, b_{\nu} \rightarrow a(\nu \rightarrow \infty) \Rightarrow \liminf _{\nu \rightarrow \infty} \frac{\left|f\left(a_{\nu}\right)-f\left(b_{\nu}\right)\right|}{\left|a_{\nu}-b_{\nu}\right|}>0 .
$$

3. The $\mathcal{W L}$ class of regularity conditions. In this section we describe some class of regularity conditions and we prove that in o-minimal geometry they are in some sense invariant under definable, locally Lipschitz, weakly bi-Lipschitz homeomorphisms. As shown in the next sections, this class includes the Whitney (B) condition and the Verdier condition.

From now on we fix, on the ordered field $\mathbb{R}$, an o-minimal structure $\mathcal{D}$ admitting definable $C^{q}$ cell decompositions ( $q \geq 1$ is also fixed). In the whole paper, "definable" means "definable in $\mathcal{D}$ ".

Theorem 3.1. Let $p \in \mathbb{N}, p \geq 1$. For any finite family of definable sets $A, B_{1}, \ldots, B_{p} \subset A \subset \mathbb{R}^{n}$, there exists a finite definable $C^{q}$ stratification of $A$ compatible with $B_{i}, i=1, \ldots, p$.

Proof. This easily follows from the $C^{q}$ Cell Decomposition Theorem (see [DM, 4.2]).

Let $\mathcal{Q}$ be a condition on pairs $(\Lambda, \Gamma)$ at points $x \in \Gamma$, where $\Lambda, \Gamma \subset \mathbb{R}^{n}$ are $C^{q}$ submanifolds with $\Gamma \subset \bar{\Lambda} \backslash \Lambda$. Sometimes we will refer to $\mathcal{Q}$ as a regularity condition.

Definition 3.2. We say that the condition $\mathcal{Q}$ is local if for any open neighbourhood $U$ of any $x \in \Gamma$ the pair $(\Lambda, \Gamma)$ satisfies $\mathcal{Q}$ at $x$ if and only if $(\Lambda \cap U, \Gamma \cap U)$ satisfies $\mathcal{Q}$ at $x$. 
We will be considering only local conditions. We adopt the following notation:

$\mathcal{W}^{\mathcal{Q}}(\Lambda, \Gamma, x) \equiv$ the condition $\mathcal{Q}$ is satisfied for the pair $(\Lambda, \Gamma)$ at $x \in \Gamma$,

$\mathcal{W}^{\mathcal{Q}}(\Lambda, \Gamma) \equiv \mathcal{W}^{\mathcal{Q}}(\Lambda, \Gamma, x)$ for each $x \in \Gamma$,

$\neg \mathcal{W}^{\mathcal{Q}}(\Lambda, \Gamma, x) \equiv$ the negation of $\mathcal{W}^{Q}(\Lambda, \Gamma, x)$.

Definition 3.3. We say that $\mathcal{Q}$ is definable if for any definable $C^{q}$ submanifolds $\Gamma, \Lambda \subset \mathbb{R}^{n}$ such that $\Gamma \subset \bar{\Lambda} \backslash \Lambda$, the set

$$
\left\{x \in \Gamma: \mathcal{W}^{\mathcal{Q}}(\Lambda, \Gamma, x)\right\}
$$

is definable.

Definition 3.4. Let $\mathcal{Q}$ be a definable condition. We say that $\mathcal{Q}$ is $g e$ neric if for any definable $C^{q}$ submanifolds $\Lambda, \Gamma \subset \mathbb{R}^{n}$ with $\Gamma \subset \bar{\Lambda} \backslash \Lambda$, the set

$$
\left\{x \in \Gamma: \neg \mathcal{W}^{\mathcal{Q}}(\Lambda, \Gamma, x)\right\}
$$

is nowhere dense in $\Gamma$.

We are interested in $C^{q}$ stratifications satisfying a condition $\mathcal{Q}$.

Definition 3.5. Let $\mathfrak{X}_{A}$ be a $\mathcal{C}^{q}$ stratification of $A$ and $\mathcal{Q}$ a condition. We say that $\mathfrak{X}_{A}$ is a stratification with condition $\mathcal{Q}$ (or a $\mathcal{Q}$-stratification) if for each pair $\Lambda, \Gamma \subset \mathfrak{X}_{A}$,

$$
\Gamma \subset \bar{\Lambda} \backslash \Lambda \Rightarrow \mathcal{W}^{\mathcal{Q}}(\Lambda, \Gamma)
$$

Theorem 3.6 (Łojasiewicz-Stasica-Wachta). Let $\mathcal{Q}$ be a definable, generic condition. Then for any finite family $\left\{A_{j}\right\}_{j \in I}$ of definable subsets of $\mathbb{R}^{n}$, there exists a finite definable $C^{q}$ stratification $\mathfrak{X}_{\mathbb{R}^{n}}$ of $\mathbb{R}^{n}$ with condition $\mathcal{Q}$, compatible with $\left\{A_{j}\right\}_{j \in I}$.

Proof. In [ $\mathrm{ESW}$, Prop. 2] a proof was given in the subanalytic case. It suffices to observe that the same argument works in the general definable case.

Corollary 3.7. Let $\mathcal{Q}$ be a definable, generic condition. Given a definable $C^{q}$ stratification $\mathfrak{X}_{A}$ of a definable set $A \subset \mathbb{R}^{n}$, there exists a finite definable $C^{q}$ stratification $\mathfrak{X}_{A}^{\prime}$ of $A$ with condition $\mathcal{Q}$ such that $\mathfrak{X}_{A}^{\prime}$ is compatible with $\mathfrak{X}_{A}$ and moreover

$$
\left\{\Gamma^{\prime} \in \mathfrak{X}_{A}: \operatorname{dim} \Gamma^{\prime}=\operatorname{dim} A\right\}=\left\{\Gamma \in \mathfrak{X}_{A}: \operatorname{dim} \Gamma=\operatorname{dim} A\right\} .
$$

Proof. Observe that using the downward induction from [ $\mathrm{ESW}$ ] and modifying the definable $C^{q}$ stratification to one satisfying condition $\mathcal{Q}$, all we need to do is to substratify those strata of $\mathfrak{X}_{A}$ of $\operatorname{dimension}<\operatorname{dim} A$.

Definition 3.8. We say that a condition $\mathcal{Q}$ is $C^{q}$ invariant (or invariant under $C^{q}$ diffeomorphisms) if for any $C^{q}$ diffeomorphism $\phi: U \rightarrow W$ of open 
subsets $U, W \subset \mathbb{R}^{n}$ and any $C^{q}$ submanifolds $\Lambda, \Gamma \subset U$ such that $\Gamma \subset \bar{\Lambda} \backslash \Lambda$, and for any point $x \in \Gamma$,

$$
\mathcal{W}^{Q}(\Lambda, \Gamma, x) \Leftrightarrow \mathcal{W}^{\mathcal{Q}}(\phi(\Lambda), \phi(\Gamma), \phi(x)) .
$$

The class of conditions that we are to describe consists of conditions that are definable, generic and invariant under definable $C^{q}$ diffeomorphisms. Additionally, two more features are required.

Definition 3.9. We say that the condition $\mathcal{Q}$ has the projection property with respect to weakly Lipschitz mappings of class $C^{q}$ if for any mapping $f: A \rightarrow \mathbb{R}^{m}$ weakly Lipschitz of class $C^{q}$ on a $C^{q}$ stratification $\mathfrak{X}_{A}$ of a set $A \subset \mathbb{R}^{n}$, we have

$$
\mathfrak{X}_{\text {graph } f}\left(\mathfrak{X}_{A}\right) \text { is a } \mathcal{Q} \text {-stratification } \Rightarrow \mathfrak{X}_{A} \text { is a } \mathcal{Q} \text {-stratification. }
$$

Notice that this condition is equivalent to the following one:

For any subset $E \subset \mathbb{R}^{n} \times \mathbb{R}^{m}$ and its $C^{q}$ stratification $\mathfrak{X}_{E}$ such that $\left.\pi_{1}\right|_{E}: E \rightarrow \mathbb{R}^{n}$ is a homeomorphic embedding and for each $\Gamma \in \mathfrak{X}_{E}$, $\left.\pi_{1}\right|_{\Gamma}: \Gamma \rightarrow \mathbb{R}^{n}$ is a $C^{q}$ embedding and $\left(\left.\pi_{2}\right|_{E}\right) \circ\left(\left.\pi_{1}\right|_{E}\right)^{-1}$ is weakly Lipschitz of class $C^{q}$ on $\pi_{1} \mathfrak{X}_{E}$, we have

$$
\mathfrak{X}_{E} \text { is a } \mathcal{Q} \text {-stratification } \Rightarrow \pi_{1} \mathfrak{X}_{E} \text { is a } \mathcal{Q} \text {-stratification, }
$$

where $\pi_{1}: \mathbb{R}^{n} \times \mathbb{R}^{m} \rightarrow \mathbb{R}^{n}$ and $\pi_{2}: \mathbb{R}^{n} \times \mathbb{R}^{m} \rightarrow \mathbb{R}^{m}$ denote the natural projections.

Remark 3.10. By Propositions 2.9 and 2.11 the condition that the mapping $\left(\left.\pi_{2}\right|_{E}\right) \circ\left(\left.\pi_{1}\right|_{E}\right)^{-1}$ is weakly Lipschitz of class $C^{q}$ on $\pi_{1} \mathfrak{X}_{E}$ is equivalent to $\left.\pi_{1}\right|_{E}$ being weakly bi-Lipschitz of class $C^{q}$ on $\mathfrak{X}_{E}$.

The following proposition is obvious.

Proposition 3.11. Let $\mathcal{Q}$ be a condition having the projection property with respect to weakly Lipschitz mappings of class $C^{q}$. Let $f: A \rightarrow \mathbb{R}^{m}$ be weakly Lipschitz of class $C^{q}$ on a stratification $\mathfrak{X}_{A}$ of a set $A \subset \mathbb{R}^{n}$. Then for any $C^{q}$ submanifolds $\Gamma, \Lambda \subset \operatorname{graph} f$ such that $\Gamma \subset \bar{\Lambda} \backslash \Lambda, \operatorname{dim} \Gamma<\operatorname{dim} \Lambda\left(^{1}\right)$ and $\{\Lambda, \Gamma\}$ is compatible with the stratification $\mathfrak{X}_{\text {graph } f}\left(\mathfrak{X}_{A}\right)$,

$$
\mathcal{W}^{\mathcal{Q}}(\Lambda, \Gamma) \Rightarrow \mathcal{W}^{\mathcal{Q}}\left(\pi_{1}(\Lambda), \pi_{1}(\Gamma)\right) .
$$

Definition 3.12. We say that the condition $\mathcal{Q}$ has the lifting property with respect to locally Lipschitz mappings of class $C^{q}$ if for any two $C^{q}$ submanifolds $\Lambda, \Gamma \subset \mathbb{R}^{n}$ such that $\Gamma \subset \bar{\Lambda} \backslash \Lambda$, for any locally Lipschitz mapping $f: \Lambda \cup \Gamma \rightarrow \mathbb{R}^{m}$ such that the restrictions $\left.f\right|_{\Lambda},\left.f\right|_{\Gamma}$ are of class $C^{q}$, and for any $C^{q}$ submanifolds $M, N \subset \mathbb{R}^{n}$ such that $N \subset \bar{M} \backslash M$ and $\{M, N\}$ is

$\left({ }^{1}\right)$ The inequality $\operatorname{dim} \Gamma<\operatorname{dim} \Lambda$ is required for $\{\Lambda, \Gamma\}$ to be a stratification of $\Lambda \cup \Gamma$. In the definable case the inequality follows from $\Gamma \subset \bar{\Lambda} \backslash \Lambda$. 
compatible with $\{\Lambda, \Gamma\}$, we have

$$
\mathcal{W}^{\mathcal{Q}}(M, N) \wedge \mathcal{W}^{\mathcal{Q}}\left(\left.\operatorname{graph} f\right|_{\Lambda},\left.\operatorname{graph} f\right|_{\Gamma}\right) \Rightarrow \mathcal{W}^{\mathcal{Q}}\left(\left.\operatorname{graph} f\right|_{M},\left.\operatorname{graph} f\right|_{N}\right) .
$$

Equivalently, $\mathcal{Q}$ has the lifting property with respect to locally Lipschitz mappings of class $C^{q}$ if for any two $C^{q}$ submanifolds $\Lambda, \Gamma \subset \mathbb{R}^{n} \times \mathbb{R}^{m}$ such that $\Gamma \subset \bar{\Lambda} \backslash \Lambda$ and $\left.\pi_{1}\right|_{\Lambda \cup \Gamma}$ is a homeomorphic embedding, $\left.\pi_{1}\right|_{\Lambda},\left.\pi_{1}\right|_{\Gamma}$ are $C^{q}$ embeddings and the mapping $\left.\pi_{2}\right|_{\Lambda \cup \Gamma} \circ\left(\left.\pi_{1}\right|_{\Lambda \cup \Gamma}\right)^{-1}: \pi_{1}(\Lambda) \cup \pi_{1}(\Gamma) \rightarrow$ $\mathbb{R}^{m}$ is locally Lipschitz, the following holds true: for any $C^{q}$ submanifolds $M, N \subset \mathbb{R}^{n}$ such that $N \subset \bar{M} \backslash M$,

(i) $\mathcal{W}^{\mathcal{Q}}(M, N), \mathcal{W}^{\mathcal{Q}}(\Lambda, \Gamma), M \subset \pi_{1}(\Lambda), N \subset \pi_{1}(\Gamma)$ $\Rightarrow \mathcal{W}^{\mathcal{Q}}\left(\left(M \times \mathbb{R}^{m}\right) \cap \Lambda,\left(N \times \mathbb{R}^{m}\right) \cap \Gamma\right)$,

(ii) $\mathcal{W}^{\mathcal{Q}}(M, N), M, N \subset \pi_{1}(\Lambda) \Rightarrow \mathcal{W}^{\mathcal{Q}}\left(\left(M \times \mathbb{R}^{m}\right) \cap \Lambda,\left(N \times \mathbb{R}^{m}\right) \cap \Lambda\right)$,

(iii) $\mathcal{W}^{\mathcal{Q}}(M, N), M, N \subset \pi_{1}(\Gamma) \Rightarrow \mathcal{W}^{\mathcal{Q}}\left(\left(M \times \mathbb{R}^{m}\right) \cap \Gamma,\left(N \times \mathbb{R}^{m}\right) \cap \Gamma\right)$.

REMARK 3.13. If a condition $\mathcal{Q}$ is $C^{q}$ invariant, then the implications (ii) and (iii) are always satisfied.

Definition 3.14. We say that a condition $\mathcal{Q}$ is a $\mathcal{W L}$ condition of class $C^{q}$ if it is

- definable;

- generic;

- invariant under definable $C^{q}$ diffeomorphisms;

- has the projection property with respect to weakly Lipschitz mappings of class $C^{q}$;

- has the lifting property with respect to locally Lipschitz mappings of class $C^{q}$.

Conditions of type $\mathcal{W L}$ are invariant under definable locally Lipschitz, weakly bi-Lipschitz homeomorphisms in the following sense:

TheOREM 3.15 (Invariance of $\mathcal{W L}$ conditions under definable, locally Lipschitz, weakly bi-Lipschitz homeomorphisms). Let $\mathcal{Q}$ be a $\mathcal{W} \mathcal{L}$ condition of class $C^{q}$. Let $A \subset \mathbb{R}^{n}$ be a definable set and let $f: A \rightarrow \mathbb{R}^{m}$ be a definable homeomorphic embedding, weakly bi-Lipschitz of class $C^{q}$ on a definable $C^{q}$ stratification $\mathfrak{X}_{A}$ of $A$. Assume that for any two submanifolds $\Lambda, \Gamma \in \mathfrak{X}_{A}$ such that $\Gamma \subset \bar{\Lambda} \backslash \Lambda$, the mapping $\left.f\right|_{\Lambda \cup \Gamma}$ is locally Lipschitz. Then there exists a definable $C^{q}$ stratification $\mathfrak{X}_{A}^{\prime}$ of $A$, compatible with $\mathfrak{X}_{A}$, such that

$$
\left\{\Gamma \in \mathfrak{X}_{A}: \operatorname{dim} \Gamma=\operatorname{dim} A\right\}=\left\{\Gamma^{\prime} \in \mathfrak{X}_{A}^{\prime}: \operatorname{dim} \Gamma^{\prime}=\operatorname{dim} A\right\}
$$

and the condition $\mathcal{Q}$ is invariant with respect to the pair $\left(f, \mathfrak{X}_{A}^{\prime}\right)$ in the following sense: for any definable $C^{q}$ submanifolds $M, N \subset A$ such that $N \subset \bar{M} \backslash M$ and $\{M, N\}$ is compatible with the stratification $\mathfrak{X}_{A}^{\prime}$,

$$
\mathcal{W}^{\mathcal{Q}}(M, N) \Rightarrow \mathcal{W}^{\mathcal{Q}}(f(M), f(N)) .
$$


Proof. Consider graph $f \subset \mathbb{R}^{n} \times \mathbb{R}^{m}$ and its definable $C^{q}$ stratification

$$
\mathfrak{X}_{\text {graph } f}\left(\mathfrak{X}_{A}\right)=\left\{\text { graph }\left.f\right|_{\Gamma}: \Gamma \in \mathfrak{X}_{A}\right\} .
$$

By Corollary 3.7 we find a definable $C^{q}$ substratification $\mathfrak{X}_{\text {graph } f}^{\mathcal{Q}}\left(\mathfrak{X}_{A}\right)$ of graph $f$ with condition $\mathcal{Q}$ that is compatible with the family $\mathfrak{X}_{\text {graph } f}\left(\mathfrak{X}_{A}\right)$ and moreover

$$
\left\{\Gamma^{\prime} \in \mathfrak{X}_{\text {graph } f}^{\mathcal{Q}}\left(\mathfrak{X}_{A}\right): \operatorname{dim} \Gamma^{\prime}=\operatorname{dim} A\right\}=\left\{\Gamma \in \mathfrak{X}_{\operatorname{graph} f}\left(\mathfrak{X}_{A}\right): \operatorname{dim} \Gamma=\operatorname{dim} A\right\} .
$$

We will show that

$$
\mathfrak{X}_{A}^{\prime}=\left\{\pi_{1}(\Lambda): \Lambda \in \mathfrak{X}_{\text {graph } f}^{\mathcal{Q}}\left(\mathfrak{X}_{A}\right)\right\}
$$

is the required stratification.

Of course $\mathfrak{X}_{A}^{\prime}$ is a definable $C^{q}$ stratification of $A$, compatible with $\mathfrak{X}_{A}$ and such that

$$
\left\{\Gamma \in \mathfrak{X}_{A}: \operatorname{dim} \Gamma=\operatorname{dim} A\right\}=\left\{\Lambda \in \mathfrak{X}_{A}^{\prime}: \operatorname{dim} \Lambda=\operatorname{dim} A\right\} .
$$

By the projection property of the condition $\mathcal{Q}$ with respect to weakly Lipschitz mappings, $\mathfrak{X}_{A}^{\prime}$ is a $\mathcal{Q}$-stratification.

Observe that for any two strata $\Lambda, \Gamma \in \mathfrak{X}_{A}^{\prime}$ with $\Gamma \subset \bar{\Lambda} \backslash \Lambda$, the mapping $\left.f\right|_{\Lambda \cup \Gamma}$ is still locally Lipschitz, by the compatibility of $\mathfrak{X}_{A}^{\prime}$ with $\mathfrak{X}_{A}$.

For the same reason $f \mathfrak{X}_{A}^{\prime}$ is compatible with $f \mathfrak{X}_{A}$. Therefore, from Proposition 2.8 we see that $f$ is still weakly bi-Lipschitz of class $C^{q}$ on $\mathfrak{X}_{A}^{\prime}$.

Consider now two definable $C^{q}$ submanifolds $M, N$ in $\mathbb{R}^{n}$ with $N \subset \bar{M} \backslash M$ that are compatible with the stratification $\mathfrak{X}_{A}^{\prime}$ and $\mathcal{W}^{\mathcal{Q}}(M, N)$ holds. Then two cases are possible:

I. There exists a submanifold $\Lambda \in \mathfrak{X}_{A}^{\prime}$ such that $M, N \subset \Lambda$. As $\left.f\right|_{\Lambda}$ is a definable $C^{q}$ embedding, by the invariance of $\mathcal{Q}$ under definable $C^{q}$ diffeomorphisms we get $\mathcal{W}^{\mathcal{Q}}(f(M), f(N))$.

II. There exist two different $\Lambda, \Gamma \in \mathfrak{X}_{A}^{\prime}$ such that $N \subset \Gamma, M \subset \Lambda$. Then $\Gamma \subset \bar{\Lambda} \backslash \Lambda$ and $\left.f\right|_{\Lambda \cup \Gamma}$ is still locally Lipschitz.

In this case, $\mathcal{W}^{\mathcal{Q}}$ (graph $\left.f\right|_{\Lambda}$, graph $\left.f\right|_{\Gamma}$ ) holds by the construction of $\mathfrak{X}_{\text {graph } f}^{\mathcal{Q}}\left(\mathfrak{X}_{A}\right)$, so as $\mathcal{Q}$ has the lifting property under locally Lipschitz mappings of class $C^{q}$, we get

$$
\mathcal{W}^{\mathcal{Q}}\left(\left.\operatorname{graph} f\right|_{M},\left.\operatorname{graph} f\right|_{N}\right) \text {. }
$$

On the other hand, $f^{-1}$ is weakly Lipschitz on the definable $C^{q}$ stratification $\{f(\Lambda), f(\Gamma)\}$ and thus on $\{f(M), f(N)\}$. Observe that

$$
\Phi\left(\left.\operatorname{graph} f\right|_{M}\right)=\left.\operatorname{graph} f^{-1}\right|_{f(M)}, \quad \Phi\left(\left.\operatorname{graph} f\right|_{N}\right)=\left.\operatorname{graph} f^{-1}\right|_{f(N)},
$$

where $\Phi: \mathbb{R}^{n} \times \mathbb{R}^{m} \ni(x, y) \mapsto(y, x) \in \mathbb{R}^{m} \times \mathbb{R}^{n}$. Therefore, by the projection property of $\mathcal{Q}$ with respect to weakly Lipschitz mappings we have

$$
\mathcal{W}^{\mathcal{Q}}\left(\pi_{2}\left(\left.\operatorname{graph} f\right|_{M}\right), \pi_{2}\left(\left.\operatorname{graph} f\right|_{N}\right)\right) \text {. }
$$


But $\pi_{2}\left(\left.\operatorname{graph} f\right|_{M}\right)=f(M)$ and $\left.\pi_{2}\left(\left.\operatorname{graph} f\right|_{N}\right)\right)=f(N)$, which completes the proof.

REMARK 3.16. As a corollary from the proof of Theorem 3.15 we find that the definable $C^{q}$ stratifications $\mathfrak{X}_{A}^{\prime}$ and $\mathfrak{X}_{f(A)}^{\prime}=\left\{f(\Gamma): \Gamma \in \mathfrak{X}_{A}^{\prime}\right\}$ are $\mathcal{Q}$-stratifications.

4. The Whitney (B) condition as a $\mathcal{W L}$ condition. In this section we will prove that the Whitney (B) condition is of type $\mathcal{W L}$ of class $C^{q}$, $q \geq 1$.

Definition 4.1. Let $N, M$ be $C^{q}$ submanifolds of $\mathbb{R}^{n}$ such that $N \subset$ $\bar{M} \backslash M, \operatorname{dim} N<\operatorname{dim} M$ and let $a \in N$.

We say that the pair of strata $(M, N)$ satisfies the Whitney (B) condition at the point a (notation: $\mathcal{W}^{B}(M, N, a)$ ) if for any sequences $\left\{a_{\nu}\right\}_{\nu \in \mathbb{N}} \subset N$, $\left\{b_{\nu}\right\}_{\nu \in \mathbb{N}} \subset M$ both converging to $a$ and such that the sequence of the secant lines $\left\{\mathbb{R}\left(a_{\nu}-b_{\nu}\right)\right\}_{\nu \in \mathbb{N}}$ converges to a line $L \subset \mathbb{R}^{n}$ in $\mathbb{P}_{n-1}$ and the sequence of the tangent spaces $\left\{T_{b_{\nu}} M\right\}_{\nu \in \mathbb{N}}$ converges to a subspace $T \subset \mathbb{R}^{n}$ in $\mathbb{G}_{\operatorname{dim} M, n}$, always $L \subset T$.

If $\mathcal{W}^{B}(M, N, a)$ for any point $a \in N$, we write $\mathcal{W}^{B}(M, N)$.

TheOrem 4.2. The Whitney (B) condition is definable and generic.

Proof. The proof in [TL2] for the structure $\left(\mathbb{R},+, \cdot, \exp ,(r)_{r \in \mathbb{R}}\right)$ remains valid for arbitrary o-minimal structures on $(\mathbb{R},<,+, \cdot)$. See also [DM].

Definition 4.3. A definable $C^{q}$ stratification $\mathfrak{X}_{A}$ of a definable set $A \subset \mathbb{R}^{n}$ is called a Whitney stratification if for any pair of strata $\Gamma, \Lambda \in \mathfrak{X}_{A}$ such that $\Gamma \subset \bar{\Lambda} \backslash \Lambda$, the condition $\mathcal{W}^{B}(\Lambda, \Gamma)$ is satisfied.

REMARK 4.4. The Whitney (B) condition is invariant under $C^{1}$ diffeomorphisms (see [Tro]).

In order to show that the Whitney (B) condition has the projection property with respect to weakly Lipschitz mappings of class $C^{q}$, it suffices to prove the following theorem.

TheOREM 4.5. Let $\Lambda, \Gamma \subset \mathbb{R}^{n}$ be $C^{q}$ submanifolds such that $\Gamma \subset \bar{\Lambda} \backslash \Lambda$ and $\operatorname{dim} \Gamma<\operatorname{dim} \Lambda$. Consider a mapping $f: \Lambda \cup \Gamma \rightarrow \mathbb{R}^{m}$ weakly Lipschitz of class $C^{q}$ on the stratification $\{\Lambda, \Gamma\}$. Then

$$
\mathcal{W}^{B}\left(\text { graph }\left.f\right|_{\Lambda} \text {, graph }\left.f\right|_{\Gamma}\right) \Rightarrow \mathcal{W}^{B}(\Lambda, \Gamma) .
$$

Proof. Let $a \in \Gamma$ and $\left\{a_{k}\right\}_{k \in \mathbb{N}} \subset \Gamma,\left\{b_{k}\right\}_{k \in \mathbb{N}} \subset \Lambda$ be sequences converging to $a$ and such that

$$
\mathbb{R}\left(a_{k}-b_{k}\right) \rightarrow L, \quad T_{b_{k}} \Lambda \rightarrow T, \quad k \rightarrow \infty,
$$

with some $L \in \mathbb{P}_{n-1}$ and $T \in \mathbb{G}_{\operatorname{dim} \Lambda, n}$. Then

$$
\left(a_{k}, f\left(a_{k}\right)\right) \rightarrow(a, f(a)), \quad\left(b_{k}, f\left(b_{k}\right)\right) \rightarrow(a, f(a)), \quad k \rightarrow \infty .
$$


Without loss of generality we may assume that for $k \rightarrow \infty$ we have

$$
\mathbb{R}\left(\left(a_{k}, f\left(a_{k}\right)\right)-\left(b_{k}, f\left(b_{k}\right)\right)\right) \rightarrow L^{\prime},\left.\quad T_{\left(b_{k}, f\left(b_{k}\right)\right)} \operatorname{graph} f\right|_{\Lambda} \rightarrow T^{\prime}
$$

with some $L^{\prime} \in \mathbb{P}_{n+m-1}, T^{\prime} \in \mathbb{G}_{\operatorname{dim} \Lambda, n+m}$. Since $\mathcal{W}^{B}\left(\left.\operatorname{graph} f\right|_{\Lambda}\right.$, graph $\left.\left.f\right|_{\Gamma}\right)$, we have $L^{\prime} \subset T^{\prime}$. Because $f$ is weakly Lipschitz on $\{\Lambda, \Gamma\}$, it follows that

$$
\pi_{1}\left(L^{\prime}\right)=L \text {. }
$$

On the other hand, as $\left.f\right|_{\Lambda}$ is of class $C^{q}$, for any $k \in \mathbb{N}$ we have

$$
\pi_{1}\left(\left.T_{\left(b_{k}, f\left(b_{k}\right)\right)} \operatorname{graph} f\right|_{\Lambda}\right)=T_{b_{k}} \Lambda,
$$

and hence $\pi_{1}\left(T^{\prime}\right) \subset T$ by the continuity of $\pi_{1}$. Consequently,

$$
L=\pi_{1}\left(L^{\prime}\right) \subset \pi_{1}\left(T^{\prime}\right) \subset T .
$$

Now we deal with the lifting property with respect to locally Lipschitz mappings of class $C^{q}$ for the Whitney (B) condition. It will easily follow from a more general transversal intersection theorem for the Whitney (B) condition.

TheOREM 4.6. Let $\Lambda_{i}, \Gamma_{i}(i=1,2)$ be two pairs of $C^{q}$ submanifolds in $\mathbb{R}^{n}$ such that $\Gamma_{i} \subset \bar{\Lambda}_{i} \backslash \Lambda_{i}$ and $\mathcal{W}^{B}\left(\Lambda_{i}, \Gamma_{i}\right)$. Assume that $\Lambda_{1} \cap \Lambda_{2}$ and $\Gamma_{1} \cap \Gamma_{2}$ are $C^{q}$ submanifolds such that $\Gamma_{1} \cap \Gamma_{2} \subset \overline{\Lambda_{1} \cap \Lambda_{2}}$ and for each $x_{0} \in \Gamma_{1} \cap \Gamma_{2}$ and any sequence $\left\{y_{\nu}\right\}_{\nu \in \mathbb{N}} \subset \Lambda_{1} \cap \Lambda_{2}$ converging to $x_{0}$, we have

$$
T_{y_{\nu}} \Lambda_{i} \rightarrow S_{i}(i=1,2) \Rightarrow \operatorname{dim} S_{1} \cap S_{2}=\operatorname{dim} \Lambda_{1} \cap \Lambda_{2} .
$$

Then $\mathcal{W}^{B}\left(\Lambda_{1} \cap \Lambda_{2}, \Gamma_{1} \cap \Gamma_{2}\right)\left(^{2}\right)$.

Proof. Let $x_{0} \in \Gamma_{1} \cap \Gamma_{2}$. Consider two sequences

$$
\left\{x_{n}\right\}_{n \in \mathbb{N}} \subset \Gamma_{1} \cap \Gamma_{2}, \quad\left\{y_{n}\right\}_{n \in \mathbb{N}} \subset \Lambda_{1} \cap \Lambda_{2}
$$

such that $x_{n}, y_{n} \rightarrow x_{0}$ as $n \rightarrow \infty$. Assume that $\mathbb{R}\left(x_{n}-y_{n}\right) \rightarrow L$ and that the sequences

$$
\left\{T_{y_{n}}\left(\Lambda_{1} \cap \Lambda_{2}\right)\right\}_{n \in \mathbb{N}}, \quad\left\{T_{y_{n}} \Lambda_{1}\right\}_{n \in \mathbb{N}}, \quad\left\{T_{y_{n}} \Lambda_{2}\right\}_{n \in \mathbb{N}}
$$

are convergent. Let

$$
T=\lim _{y_{n} \rightarrow x_{0}} T_{y_{n}}\left(\Lambda_{1} \cap \Lambda_{2}\right)
$$

By the assumptions,

$$
L \subset \lim _{y_{n} \rightarrow x_{0}} T_{y_{n}} \Lambda_{1} \cap \lim _{y_{n} \rightarrow x_{0}} T_{y_{n}} \Lambda_{2}=\lim _{y_{n} \rightarrow x_{0}} T_{y_{n}}\left(\Lambda_{1} \cap \Lambda_{2}\right)=T .
$$

Theorem 4.7. Let $\Lambda, \Gamma$ be $C^{q}$ submanifolds of $\mathbb{R}^{n}$ such that $\Gamma \subset \bar{\Lambda} \backslash \Lambda$. Let $f: \Lambda \cup \Gamma \rightarrow \mathbb{R}^{m}$ be a locally Lipschitz mapping such that $\left.f\right|_{\Lambda},\left.f\right|_{\Gamma}$ are of

$\left({ }^{2}\right)$ If for any $G \in\left\{\Lambda_{1}, \Gamma_{1}\right\}$ and $K \in\left\{\Lambda_{2}, \Gamma_{2}\right\}$ the submanifolds $G$ and $K$ are transversal in $\mathbb{R}^{n}$, then the conclusion of Theorem 4.6 follows from Lemma 4.2.2 in [Te]. 
class $C^{q}$. Let $M, N$ be $C^{q}$ submanifolds of $\mathbb{R}^{n}$ such that $N \subset \bar{M} \backslash M$ and $\{M, N\}$ is compatible with $\{\Lambda, \Gamma\}$. Then

$$
\mathcal{W}^{B}(M, N) \wedge \mathcal{W}^{B}\left(\left.\operatorname{graph} f\right|_{\Lambda} \text {, graph }\left.f\right|_{\Gamma}\right) \Rightarrow \mathcal{W}^{B}\left(\text { graph }\left.f\right|_{M} \text {, graph }\left.f\right|_{N}\right) .
$$

Proof. If $M, N \subset \Lambda$ or $M, N \subset \Gamma$, then $\mathcal{W}^{B}$ (graph $\left.f\right|_{M}$, graph $\left.\left.f\right|_{N}\right)$ holds, because the Whitney (B) condition is $C^{q}$ invariant. Now let $M \subset \Lambda$ and $N \subset \Gamma$. It is enough to use Theorem 4.6, where

$$
\Lambda_{1}=\left.\operatorname{graph} f\right|_{\Lambda}, \quad \Gamma_{1}=\left.\operatorname{graph} f\right|_{\Gamma}, \quad \Lambda_{2}=M \times \mathbb{R}^{m}, \quad \Gamma_{2}=N \times \mathbb{R}^{m} .
$$

The last assumption of Theorem 4.6 is satisfied, because $f$ is locally Lipschitz (use Propositions 1.6 and 1.7).

COROLlary 4.8. The Whitney (B) condition is a $\mathcal{W L}$ condition of class $C^{q}$.

COROLlary 4.9. The conclusion of Theorem 3.15 holds true for $\mathcal{Q}$ being the Whitney (B) condition.

REMARK 4.10. A similar theorem holds true in the analytic-geometric category defined in [DM], as the Whitney stratification theorem holds true in this category.

5. The Verdier condition as a $\mathcal{W L}$ condition. We start with some preparation.

Lemma 5.1. Let $V$ be a linear subspace of $\mathbb{R}^{n}, \mathbb{R}^{n}=V \oplus V^{\perp}$. Let $0<\alpha \leq 1$ be a constant and set

$$
B_{\alpha}=\left\{u \in S^{n-1}: d\left(\mathbb{R} u, V^{\perp}\right) \geq \alpha\right\} .
$$

Then there exists $C_{\alpha}>0$ such that:

(i) for any $u, w \in B_{\alpha}$ we have

$$
d\left(\mathbb{R} \pi_{V}(u), \mathbb{R} \pi_{V}(w)\right) \leq C_{\alpha} d(\mathbb{R} u, \mathbb{R} w),
$$

(ii) for any two linear subspaces $L, K \subset \mathbb{R}^{n}$ with $L \cap S^{n-1}, K \cap S^{n-1}$ $\subset B_{\alpha}$,

$$
d\left(\pi_{V}(L), \pi_{V}(K)\right) \leq C_{\alpha} d(L, K) .
$$

Proof. (i). Let $u, w \in B_{\alpha}$. Then

$$
\begin{gathered}
\left|\pi_{V}(u)\right|=\left|u-\pi_{V^{\perp}}(u)\right|=d\left(\mathbb{R} u, V^{\perp}\right) \geq \alpha, \\
\left|\pi_{V}(w)\right|=\left|w-\pi_{V^{\perp}}(w)\right|=d\left(\mathbb{R} w, V^{\perp}\right) \geq \alpha .
\end{gathered}
$$


Hence

$$
\begin{aligned}
& \left|\frac{\pi_{V}(u)}{\left|\pi_{V}(u)\right|}-\frac{\pi_{V}(w)}{\left|\pi_{V}(w)\right|}\right|=\left|\frac{\pi_{V}(u) \cdot\left|\pi_{V}(w)\right|-\pi_{V}(w) \cdot\left|\pi_{V}(u)\right|}{\left|\pi_{V}(u)\right| \cdot\left|\pi_{V}(w)\right|}\right| \\
& \quad=\left|\frac{\pi_{V}(u) \cdot\left|\pi_{V}(w)\right|-\pi_{V}(u) \cdot\left|\pi_{V}(u)\right|+\pi_{V}(u) \cdot\left|\pi_{V}(u)\right|-\pi_{V}(w) \cdot\left|\pi_{V}(u)\right|}{\left|\pi_{V}(u)\right| \cdot\left|\pi_{V}(w)\right|}\right| \\
& \quad \leq \frac{1}{\alpha^{2}}\left|\pi_{V}(u) \cdot\left(\left|\pi_{V}(w)\right|-\left|\pi_{V}(u)\right|\right)+\right| \pi_{V}(u)\left|\cdot\left(\pi_{V}(u)-\pi_{V}(w)\right)\right| \\
& \quad \leq \frac{1}{\alpha^{2}}\left(\left|\pi_{V}(u)\right| \cdot|| \pi_{V}(u)|-| \pi_{V}(w)||+\left|\pi_{V}(u)\right| \cdot\left|\pi_{V}(u)-\pi_{V}(w)\right|\right) \\
& \quad \leq \frac{2}{\alpha^{2}}\left(\left|\pi_{V}(u)-\pi_{V}(w)\right|\right)=\frac{2}{\alpha^{2}}\left|\pi_{V}(u-w)\right| \leq \frac{2}{\alpha^{2}}|u-w| .
\end{aligned}
$$

Similarly,

$$
\left|\frac{\pi_{V}(u)}{\left|\pi_{V}(u)\right|}+\frac{\pi_{V}(w)}{\left|\pi_{V}(w)\right|}\right|=\left|\frac{\pi_{V}(u)}{\left|\pi_{V}(u)\right|}-\frac{\pi_{V}(-w)}{\left|\pi_{V}(-w)\right|}\right| \leq \frac{2}{\alpha^{2}}|u+w| .
$$

Finally,

$$
\begin{aligned}
d\left(\mathbb{R} \pi_{V}(u), \mathbb{R} \pi_{V}(w)\right) & \leq \widetilde{d}\left(\mathbb{R} \pi_{V}(u), \mathbb{R} \pi_{V}(w)\right) \\
& \leq \frac{2}{\alpha^{2}} \widetilde{d}(\mathbb{R} u, \mathbb{R} v) \leq \frac{2 \sqrt{2}}{\alpha^{2}} d(\mathbb{R} u, \mathbb{R} v) .
\end{aligned}
$$

Item (ii) follows easily from (i).

We will also need the following definition of the sine of the angle between two linear subspaces.

Definition 5.2. Let $S, K \subset \mathbb{R}^{n}$ be linear subspaces. Then we define $\lambda(S, K)=\left\{\begin{aligned} \inf \left\{d(\mathbb{R} u, \mathbb{R} w): u \in S \cap S^{n-1}, w \in K \cap\right. & \left.S^{n-1}, u, w \perp S \cap K\right\} \\ 0 & \text { if } S \not \subset K \text { and } K \not \subset S, \\ & \text { if } S \subset K \text { or } K \subset S .\end{aligned}\right.$

Remark 5.3. Notice that if $S \not \subset K$ and $K \not \subset S$, then

$$
\lambda(S, K)=\delta\left(S \cap(S \cap K)^{\perp}, K \cap(S \cap K)^{\perp}\right) .
$$

Proposition 5.4. Let $k, l, n \in \mathbb{N}$ and let $S \in \mathbb{G}_{s, n}$ and $K \in \mathbb{G}_{k, n}$ be such that $\lambda(S, K)>0$. Then

(i) For any $v \in \mathbb{R}^{n}$ with $|v|=1$,

$$
d(\mathbb{R} v, S \cap K) \leq \frac{1}{\lambda(S, K)}(d(\mathbb{R} v, S)+d(\mathbb{R} v, K)) .
$$

(ii) If $R, L$ are linear subspaces in $\mathbb{R}^{n}$, then

$$
d(R \cap L, S \cap K) \leq \frac{1}{\lambda(S, K)}(d(R, S)+d(L, K)) .
$$


Proof. (i) If $v \in S \cap K$, then the desired inequality is satisfied as

$$
d(v, S \cap K)=d(v, S)=d(v, K)=0 .
$$

Assume now that $v \notin S \cap K$, which means that $d(v, S \cap K)>0$. Then

$$
\begin{aligned}
\lambda(S, K) \leq & d\left(\mathbb{R}\left(\pi_{K}(v)-\pi_{S \cap K}(v)\right), \mathbb{R}\left(\pi_{S}(v)-\pi_{S \cap K}(v)\right)\right) \\
\leq & d\left(\mathbb{R}\left(\pi_{K}(v)-\pi_{S \cap K}(v)\right), \mathbb{R}\left(v-\pi_{S \cap K}(v)\right)\right) \\
& \quad+d\left(\mathbb{R}\left(v-\pi_{S \cap K}(v)\right), \mathbb{R}\left(\pi_{S}(v)-\pi_{S \cap K}(v)\right)\right) \\
= & \frac{\left|v-\pi_{K}(v)\right|}{\left|v-\pi_{S \cap K}(v)\right|}+\frac{\left|v-\pi_{S}(v)\right|}{\left|v-\pi_{S \cap K}(v)\right|}=\frac{d(v, K)}{d(v, S \cap K)}+\frac{d(v, S)}{d(v, S \cap K)},
\end{aligned}
$$

as required.

Assertion (ii) follows trivially from (i).

Proposition 5.5. Let $p, k, s, n \in \mathbb{N}$ with $p<\min \{k, s\}$. Set

$$
\Sigma=\left\{(S, K) \in \mathbb{G}_{s, n} \times \mathbb{G}_{k, n}: \operatorname{dim}(S \cap K)=p\right\} .
$$

Then the mapping $\lambda: \Sigma \ni(S, K) \mapsto \lambda(S, K) \in[0,1]$ is continuous.

Proof. The continuity of $\lambda$ follows easily from the fact that the mapping

$$
\psi: \Sigma \ni(S, K) \mapsto S \cap K \in \mathbb{G}_{p, n}
$$

is continuous at any point $\left(S_{0}, K_{0}\right) \in \Sigma$, because Proposition 5.4(ii) implies the inequality

$$
d\left(S \cap K, S_{0} \cap K_{0}\right) \leq \frac{1}{\lambda\left(S_{0}, K_{0}\right)}\left(d\left(S, S_{0}\right)+d\left(K, K_{0}\right)\right)
$$

for any $(S, K) \in \Sigma$.

Proposition 5.6. Let $s, k, p, n \in \mathbb{N}$ and $p<\min \{k, s\}$. Let $\widetilde{\Sigma}$ be a compact subset of

$$
\Sigma=\left\{(S, K) \in \mathbb{G}_{s, n} \times \mathbb{G}_{k, n}: \operatorname{dim}(S \cap K)=p\right\} .
$$

Then $\inf \{\lambda(S, K):(S, K) \in \widetilde{\Sigma}\}>0$.

Proof. Trivial as $\lambda: \Sigma \ni(S, K) \mapsto \lambda(S, K) \in[0,1]$ is continuous and $\widetilde{\Sigma}$ is compact.

Corollary 5.7. Let $s, k, p, n \in \mathbb{N}$ with $p<\min \{k, s\}$ and let $\widetilde{\Sigma}$ be a compact subset of $\Sigma=\left\{(S, K) \in \mathbb{G}_{s, n} \times \mathbb{G}_{k, n}: \operatorname{dim}(S \cap K)=p\right\}$. Then there exists $C>0$ such that for any linear subspaces $R, L$ of $\mathbb{R}^{n}$ and for any $(S, K) \in \widetilde{\Sigma}$,

$$
d(R \cap L, S \cap K) \leq C(d(R, S)+d(L, K)) .
$$

Proof. By Propositions 5.6 and 5.4(ii) the above inequality holds with

$$
C=1 / \inf \{\lambda(S, K):(S, K) \in \widetilde{\Sigma}\} .
$$


Definition 5.8. Let $\Lambda, \Gamma$ be $C^{2}$ submanifolds of $\mathbb{R}^{n}$ with $\Gamma \subset \bar{\Lambda} \backslash \Lambda$. We say that the pair $(\Lambda, \Gamma)$ satisfies the Verdier condition at $x_{0} \in \Gamma$ (notation: $\left.\mathcal{W}^{V}\left(\Lambda, \Gamma, x_{0}\right)\right)$ if there exists an open neighbourhood $U_{x_{0}}$ of $x_{0}$ in $\mathbb{R}^{n}$ and $C_{x_{0}}>0$ such that

$$
\forall x \in \Gamma \cap U_{x_{0}} \forall y \in \Lambda \cap U_{x_{0}} \quad d\left(T_{x} \Gamma, T_{y} \Lambda\right) \leq C_{x_{0}}|x-y| .
$$

We say that $(\Lambda, \Gamma)$ satisfies the Verdier condition (notation $\mathcal{W}^{V}(\Lambda, \Gamma)$ ) if $\mathcal{W}^{V}\left(\Lambda, \Gamma, x_{0}\right)$ for each $x_{0} \in \Gamma$.

In 1998 Ta Le Loi proved that

TheOREM 5.9. The Verdier condition is definable and generic.

Proof. See [TL1] (cf. [ЕSW] and [DW]).

REMARK 5.10. As explained in [Ver, the Verdier condition is invariant under definable $C^{q}$ diffeomorphisms, $q \geq 2$. However, it is not $C^{1}$ invariant, as was shown in $[\mathrm{BT}]$.

Now we prove that the Verdier condition has the projection property with respect to weakly Lipschitz mappings of class $C^{q}$, where $q \geq 2$.

THEOREM 5.11. Let $q \geq 2$ and let $\Lambda, \Gamma$ be $C^{q}$ submanifolds of $\mathbb{R}^{n} \times \mathbb{R}^{m}$ with $\Gamma \subset \bar{\Lambda} \backslash \Lambda$ and $\operatorname{dim} \Gamma<\operatorname{dim} \Lambda\left({ }^{3}\right)$. Suppose that $\left.\pi_{1}\right|_{\Lambda \cup \Gamma}$ is a homeomorphic embedding such that $\left.\pi_{1}\right|_{\Lambda},\left.\pi_{1}\right|_{\Gamma}$ are $C^{q}$ embeddings ${ }^{\left({ }^{4}\right)}$, Assume that $\left.\pi_{2}\right|_{\Lambda \cup \Gamma} \circ\left(\left.\pi_{1}\right|_{\Lambda \cup \Gamma}\right)^{-1}$ is weakly Lipschitz on the $C^{q}$ stratification $\left\{\pi_{1}(\Lambda), \pi_{1}(\Gamma)\right\}$. Then

$$
\mathcal{W}^{V}(\Lambda, \Gamma) \Rightarrow \mathcal{W}^{V}\left(\pi_{1}(\Lambda), \pi_{1}(\Gamma)\right)
$$

Proof. Put $x^{\prime}=\left.\pi_{2}\right|_{\Lambda \cup \Gamma} \circ\left(\left.\pi_{1}\right|_{\Lambda \cup \Gamma}\right)^{-1}(x)$ for any $x \in \pi_{1}(\Lambda \cup \Gamma)$.

Let $x_{0} \in \pi_{1}(\Gamma)$. After a suitable $C^{2}$ change of coordinates in a neighbourhood of $\left(x_{0}, x_{0}^{\prime}\right)$ we can assume that $\pi_{1}(\Gamma)=\mathbb{R}^{k} \times\{0\}^{n-k}$ and $\Gamma=$ $\pi_{1}(\Gamma) \times\{0\}^{m}=\mathbb{R}^{k} \times\{0\}^{n+m-k}=\mathbb{R}^{k}$. Then $x^{\prime}=0$ for any $x \in \pi_{1}(\Gamma)$.

There exists an open neighbourhood $U_{\left(x_{0}, 0\right)}$ of $\left(x_{0}, 0\right)$ in $\mathbb{R}^{n} \times \mathbb{R}^{m}$ and a constant $C_{\left(x_{0}, 0\right)}>0$ such that

$$
d\left(T_{(x, 0)} \Gamma, T_{\left(y, y^{\prime}\right)} \Lambda\right) \leq C_{\left(x_{0}, 0\right)}\left|(x, 0)-\left(y, y^{\prime}\right)\right|
$$

for all $(x, 0) \in \Gamma \cap U_{\left(x_{0}, 0\right)}$ and $\left(y, y^{\prime}\right) \in \Lambda \cap U_{\left(x_{0}, 0\right)}$.

Since $\left.\pi_{2}\right|_{\Lambda \cup \Gamma} \circ\left(\left.\pi_{1}\right|_{\Lambda \cup \Gamma}\right)^{-1}$ is weakly Lipschitz on the stratification $\left\{\pi_{1}(\Lambda), \pi_{1}(\Gamma)\right\}$, there is a neighbourhood $U_{x_{0}}$ of $x_{0}$ in $\mathbb{R}^{n}$ and a constant $L_{x_{0}}>0$ such that

$$
\frac{\left|0-y^{\prime}\right|}{|x-y|} \leq L_{x_{0}}
$$

for all $x \in \pi_{1}(\Gamma) \cap U_{x_{0}}$ and $y \in \pi_{1}(\Lambda) \cap U_{x_{0}}$.

$\left({ }^{3}\right)$ Again we have to assume that $\{\Lambda, \Gamma\}$ is a $C^{q}$ stratification of $\Lambda \cup \Gamma$.

$\left({ }^{4}\right)$ As before, $\pi_{1}: \mathbb{R}^{n} \times \mathbb{R}^{m} \rightarrow \mathbb{R}^{n}$ and $\pi_{2}: \mathbb{R}^{n} \times \mathbb{R}^{m} \rightarrow \mathbb{R}^{m}$ are the natural projections. 
Without loss of generality we may assume that $U_{x_{0}}=U_{\left(x_{0}, 0\right)} \cap$ $\left(\mathbb{R}^{n} \times\{0\}^{m}\right)$. Then from the above argument, for all $(x, 0) \in \Gamma \cap U_{\left(x_{0}, 0\right)}$ and $\left(y, y^{\prime}\right) \in \Lambda \cap U_{\left(x_{0}, 0\right)}$,

$$
d\left(T_{(x, 0)} \Gamma, T_{\left(y, y^{\prime}\right)} \Lambda\right) \leq C_{\left(x_{0}, 0\right)}\left|(x, 0)-\left(y, y^{\prime}\right)\right| \leq C_{\left(x_{0}, 0\right)} \sqrt{1+\left(L_{x_{0}}\right)^{2}}|x-y|,
$$

in other words

$$
d\left(\mathbb{R}^{k}, T_{\left(y, y^{\prime}\right)} \Lambda\right) \leq C_{\left(x_{0}, 0\right)} \sqrt{1+L_{x_{0}}^{2}}|x-y| .
$$

Hence, after diminishing $U_{\left(x_{0}, 0\right)}$,

$$
d\left(\mathbb{R}^{k}, T_{\left(y, y^{\prime}\right)} \Lambda\right) \leq 1-\alpha,
$$

where $0<\alpha<1$, and then denoting by $K_{y}$ the orthogonal projection of $\mathbb{R}^{k}$ onto $T_{\left(y, y^{\prime}\right)} \Lambda$, we get a $k$-dimensional subspace $K_{y}$ of $T_{\left(y, y^{\prime}\right)} \Lambda$ such that

$$
d\left(\mathbb{R}^{k}, K_{y}\right)=d\left(\mathbb{R}^{k}, T_{\left(y, y^{\prime}\right)} \Lambda\right) .
$$

Consequently, by Lemma 5.1(ii),

$$
\begin{aligned}
d\left(T_{x} \pi_{1}(\Gamma), T_{y} \pi_{1}(\Lambda)\right) & =d\left(\mathbb{R}^{k}, \pi_{1}\left(T_{\left(y, y^{\prime}\right)} \Lambda\right)\right) \\
& =d\left(\pi_{1}\left(\mathbb{R}^{k} \times\{0\}\right), \pi_{1}\left(K_{y}\right)\right) \leq C_{\alpha} d\left(\mathbb{R}^{k}, T_{\left(y, y^{\prime}\right)} \Lambda\right) \leq \widetilde{C}|x-y|,
\end{aligned}
$$

which completes the proof.

COROLlaRY 5.12. The Verdier condition has the projection property with respect to weakly Lipschitz mappings of class $C^{q}$, where $q \geq 2$.

It remains to deal with the property of lifting the Verdier condition with respect to locally Lipschitz mappings of class $C^{q}, q \geq 2$. The argument is analogous to that in the case of the Whitney (B) condition.

TheOREM 5.13. Theorem 4.6 remains true when the Whitney (B) condition is replaced by the Verdier condition, assuming that $q \geq 2$.

Proof. Let $x_{0} \in \Gamma_{1} \cap \Gamma_{2}$. There exists a neighbourhood $U_{x_{0}}$ of $x_{0}$ and a constant $C_{x_{0}}>0$ such that for all $x \in \Gamma_{i} \cap U_{x_{0}}$ and $y \in \Lambda_{i} \cap U_{x_{0}}$,

$$
d\left(T_{x} \Gamma_{i}, T_{y} \Lambda_{i}\right) \leq C_{x_{0}}|x-y|, \quad i=1,2 .
$$

CASE I. Assume that $\operatorname{dim} \Lambda_{1} \cap \Lambda_{2}=\min \left\{\operatorname{dim} \Lambda_{1}, \operatorname{dim} \Lambda_{2}\right\}=\operatorname{dim} \Lambda_{1}$ (the other case is similar). Then $\Lambda_{1} \cap \Lambda_{2}$ is open in $\Lambda_{1}$, hence $T_{y}\left(\Lambda_{1} \cap \Lambda_{2}\right)=T_{y} \Lambda_{1}$ and

$$
d\left(T_{x}\left(\Gamma_{1} \cap \Gamma_{2}\right), T_{y}\left(\Lambda_{1} \cap \Lambda_{2}\right)\right) \leq d\left(T_{x} \Gamma_{1}, T_{y} \Lambda_{1}\right) \leq C_{x_{0}}|x-y|
$$

for all $x \in \Gamma_{1} \cap \Gamma_{2} \cap U_{x_{0}}$ and $y \in \Lambda_{1} \cap \Lambda_{2} \cap U_{x_{0}}$, as desired.

CASE II. Assume now that $\operatorname{dim} \Lambda_{1} \cap \Lambda_{2}<\min \left\{\operatorname{dim} \Lambda_{1}, \operatorname{dim} \Lambda_{2}\right\}$. Let

$$
\Sigma=\left\{(S, K) \in \mathbb{G}_{\operatorname{dim} \Lambda_{1}, n} \times \mathbb{G}_{\operatorname{dim} \Lambda_{2}, n}: \operatorname{dim} S \cap K=\operatorname{dim} \Lambda_{1} \cap \Lambda_{2}\right\} .
$$


Then by the assumptions, after perhaps diminishing the neighbourhood $U_{x_{0}}$, the closure $\widetilde{\Sigma}$ of the set

$$
\left\{\left(T_{y} \Lambda_{1}, T_{y} \Lambda_{2}\right) \in \mathbb{G}_{\operatorname{dim} \Lambda_{1}, n} \times \mathbb{G}_{\operatorname{dim} \Lambda_{2}, n}: y \in \Lambda_{1} \cap \Lambda_{2} \cap U_{x_{0}}\right\}
$$

is a closed subset of $\Sigma$. By Corollary 5.7 there exists a constant $C>0$ such that for all $x \in \Gamma_{1} \cap \Gamma_{2} \cap U_{x_{0}}$ and $y \in \Lambda_{1} \cap \Lambda_{2} \cap U_{x_{0}}$ we have

$$
d\left(T_{x} \Gamma_{1} \cap T_{x} \Gamma_{2}, T_{y} \Lambda_{1} \cap T_{y} \Lambda_{2}\right) \leq C\left(d\left(T_{x} \Gamma_{1}, T_{y} \Lambda_{1}\right)+d\left(T_{x} \Gamma_{2}, T_{y} \Lambda_{2}\right)\right) .
$$

Consequently,

$$
d\left(T_{x}\left(\Gamma_{1} \cap \Gamma_{2}\right), T_{y}\left(\Lambda_{1} \cap \Lambda_{2}\right)\right) \leq C \cdot 2 C_{x_{0}} \cdot|x-y| .
$$

TheOREM 5.14. Theorem 4.7 remains true if the Whitney (B) condition is replaced by the Verdier condition, assuming that $q \geq 2$.

Proof. This follows from Theorem 5.13 in the same way as Theorem 4.7 follows from Theorem 4.6.

Corollary 5.15. The Verdier condition is a $\mathcal{W L}$ condition of class $C^{q}$, $q \geq 2$.

COROLlary 5.16. Theorem 3.15 holds true for the Verdier condition with $q \geq 2$.

Acknowledgements. I would like to express my profound gratitude to Wiesław Pawłucki for his great tutorial, friendly support and many valuable discussions.

\section{References}

[BT] H. Brodersen and D. Trotman, Whitney (b)-regularity is weaker than Kuo's ratio test for real algebraic stratifications, Math. Scand. 45 (1979), 27-34.

[DW] Z. Denkowska and K. Wachta, Une construction de la stratification sous-analytique avec la condition (w), Bull. Polish Acad. Sci. Math. 35 (1987), 401-405.

[D] L. van den Dries, Tame Topology and o-Minimal Structures, Cambridge Univ. Press, 1998.

[DM] L. van den Dries and C. Miller, Geometric categories and o-minimal structures, Duke Math. J. 84 (1996), 497-540.

[€] S. Łojasiewicz, An Introduction to Complex Analytic Geometry, Birkhäuser, 1991.

[ŁSW] S. Łojasiewicz, J. Stasica and K. Wachta, Stratifications sous-analytiques. Condition de Verdier, Bull. Polish Acad. Sci. Math. 34 (1986), 531-539.

[S] M. Shiota, Whitney triangulations of semialgebraic sets, Ann. Polon. Math. 87 (2005), 237-246.

[TL1] Ta Le Loi, Verdier and strict Thom stratifications in o-minimal structures, Illinois J. Math. 42 (1998), 347-356.

[TL2] -, Whitney stratification of sets definable in the structure $\mathbb{R}_{\exp }$, in: Banach Center Publ. 33, Inst. Math., Polish Acad. Sci., 1996, 401-409. 
[Te] B. Teissier, Variétés polaires II: Multiplicités polaires, sections planes et conditions de Whitney, in: Algebraic Geometry (La Rábida, 1981), Lecture Notes in Math. 961, Springer, 1982, 314-491.

[Tro] D. J. A. Trotman, Geometric versions of Whitney regularity for smooth stratifications, Ann. Sci. École Norm. Sup. 12 (1979), 453-463.

[Ver] J.-L. Verdier, Stratifications de Whitney et théorème de Bertini-Sard, Invent. Math. 36 (1976), 295-312.

Małgorzata Czapla

Instytut Matematyki

Uniwersytet Jagielloński

Stanisława Łojasiewicza 6

30-348 Kraków, Poland

E-mail: Malgorzata.Czapla@im.uj.edu.pl

Received 27.10.2008

and in final form 19.3.2009 
